\title{
Mit Musik geht alles besser
}

\author{
Krankenschwestern aus Taiwan \\ randomisierten 98 Krebspatienten, \\ die sich einer Chemotherapie unter- \\ ziehen mussten, in drei Gruppen. Sie \\ erhielten entweder eine einstündige, \\ entspannende Musiktherapie oder \\ eine 30-minütige Entspannungs- \\ therapie oder keines von beiden.
}

- Nach der Chemotherapie war in beiden vorbehandelten Gruppen die Ängstlichkeit weniger deutlich ausgeprägt, als in der Kontrollgruppe. Der Vergleich zwischen den beiden vorbehandelten Gruppen zeigt, dass der Effekt nach $\mathrm{Mu}-$ siktherapie deutlicher war, als nach Entspannungstherapie. Die Autoren meinen daher, dass beide Behandlungsformen den Stress einer Chemotherapie reduzieren.

\section{Kommentar}

Dieser Konklusion kann man zustimmen. Dass Entspannung Stress verhindert, leuchtet ein. Dass Entspannung auf verschiedene Weise induziert werden kann, ist ebenso plausibel. Interessant wäre, zu definieren, welche Methode dieses Ziel am ehesten erreicht. Obschon in dieser Untersuchung zwei Optionen verglichen wurden, gibt sie keine schlüssige Antwort auf diese Frage. Zwar ist der Effekt nach Musiktherapie deutlich größer, jedoch wissen wir nicht, ob das an der Therapie oder an der doppelt so langen Therapiedauer liegt.

Fazit: Vermeidbare Fehler im Studiendesign verhindern häufig, dass schlüssige Antworten auf relevante Fragen gefunden werden.

E. ERNST =

- M. F. Lin et al.

A randomised controlled trial of the effect of music therapy and verbal relaxation on chemotherapy-induced anxiety. J. Clin. Nurs. 20 (2011) 988-999 